\begin{tabular}{|c|c|}
\hline 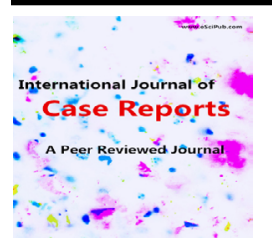 & $\begin{array}{l}\text { International Journal of Case Reports } \\
\text { (ISSN:2572-8776) }\end{array}$ \\
\hline
\end{tabular}

\title{
Dislocation of the Mandible Condyle Towards the Middle Cranial Fossa: Case Report and Review of Literature
}

MESQUITA FILHO, Paulo Mesquita1; LEAL, Elizabeth Bergamo1; MANZATO, Luciano Bambini'; DE CONTO, Ferdinando; FELIN, Gabriela Caovilla ${ }^{2}$; PONCIANO, Thales Henrique Jincziwski ${ }^{2}$

${ }^{1}$ Departament of Neurosurgery, Hospital de Clínicas, Passo Fundo, Brazil

${ }^{2}$ Departament of Oral and Maxillofacial Surgery, Hospital de Clínicas, Passo Fundo, Brazil

\section{ABSTRACT}

Introduction: Dislocation of the mandibular condyle into the

${ }^{*}$ Correspondence to Author:

middle cranial fossa is extremely rare in patients with craniofacial

Thales Henrique Jincziwski Ponciatrauma. no Campus I - Km 171 - BR 285 -

Methods: This report documents a case of an intact mandibular Bairro São José - Caixa Postal 611 condyle intrusion into the middle cranial fossa in a 17-year-old woman following a traumatic incident treated by an intraoral ap-Zip code: 99001-9470 Faculdade proach that was performed to do an open reduction by condylectomy.

How to cite this article:

Results: The causative mechanism, diagnostic features and a MESQUITA FILHO, Paulo Mesmanagement option for reduction and treatment of these cases by performing a multidisciplinary approach involving neurosurgery and oral maxillofacial surgery are presented in this report.

Conclusion: Adequate radiographic examination, including Tomog-raphy and Magnetic Resonance, is necessary to obtain the proper diagnosis and to determine an effective management.

Keywords: Trauma; Temporomandibular joint; Mandibular condylar fracture; Middle cranial fossa quita; LEAL, Elizabeth Bergamo; MANZATO, Luciano Bambini; DE CONTO, Ferdinando; FELIN, Gabriela Caovilla; PONCIANO, Thales Henrique Jincziwski. Dislocation of the Mandible Condyle Towards the Middle Cranial Fossa: Case Report and Review of Literature. International Journal of Case Reports, 2021, 5:246.

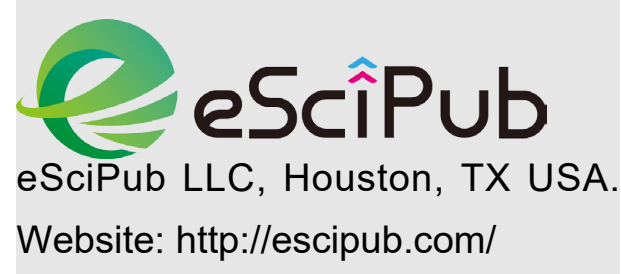




\section{INTRODUCTION}

Although fractures of the skull base are very common secondary to trauma, there are some rare types of fracture, as the lesion of the glenoid fossa of the temporal bone. Luxation of the mandible is frequent when dealing with direct blunt to the face, but dislocation of the condylar portion of the mandible towards the skull base is rare ${ }^{[1,2]}$. We present the case of a patient that had a direct trauma to the mandible, causing fracture of the articular portion of the temporal bone, and dislocation of the mandible condyle towards the middle cranial fossa.
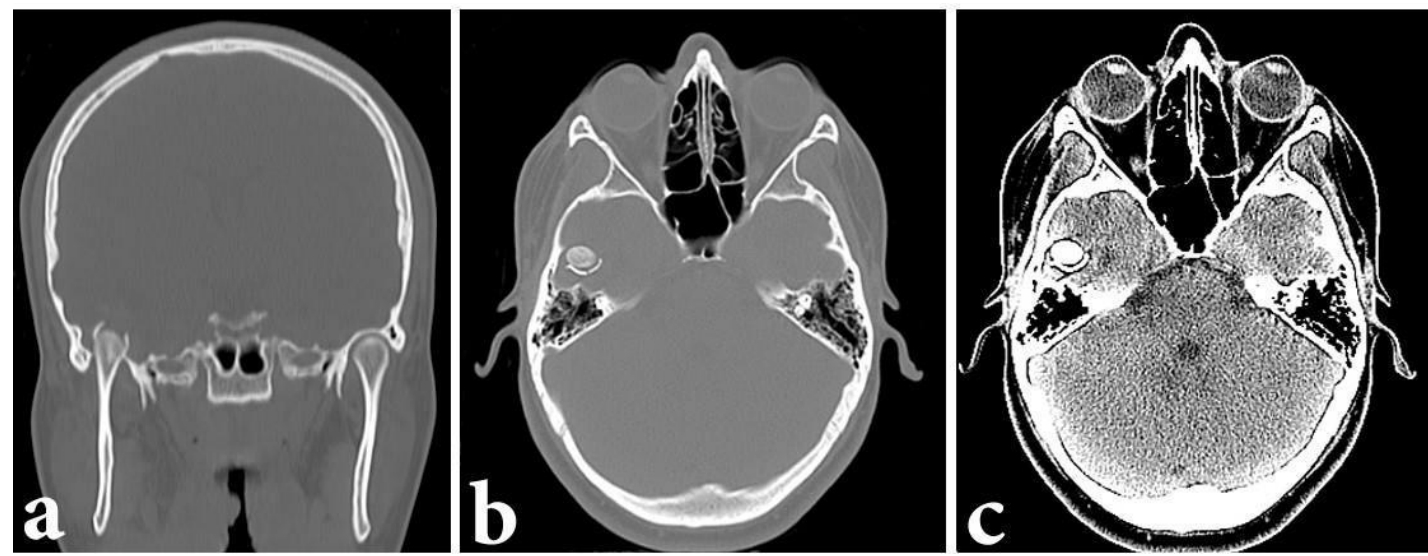

Fig. 1 (a) Coronal view: Preoperative CT scan, bony sequence, showing fracture and perforation of glenoid fossa and dislocation of condyle into the middle cranial fossa. (b) Axial view: Preoperative CT scan, bony sequence, evidencing the condyle in the middle cranial fossa. (c) Axial view: Preoperative CT scan, brain sequence, evidencing the condyle causing a small brain contusion in the right temporal lobe.
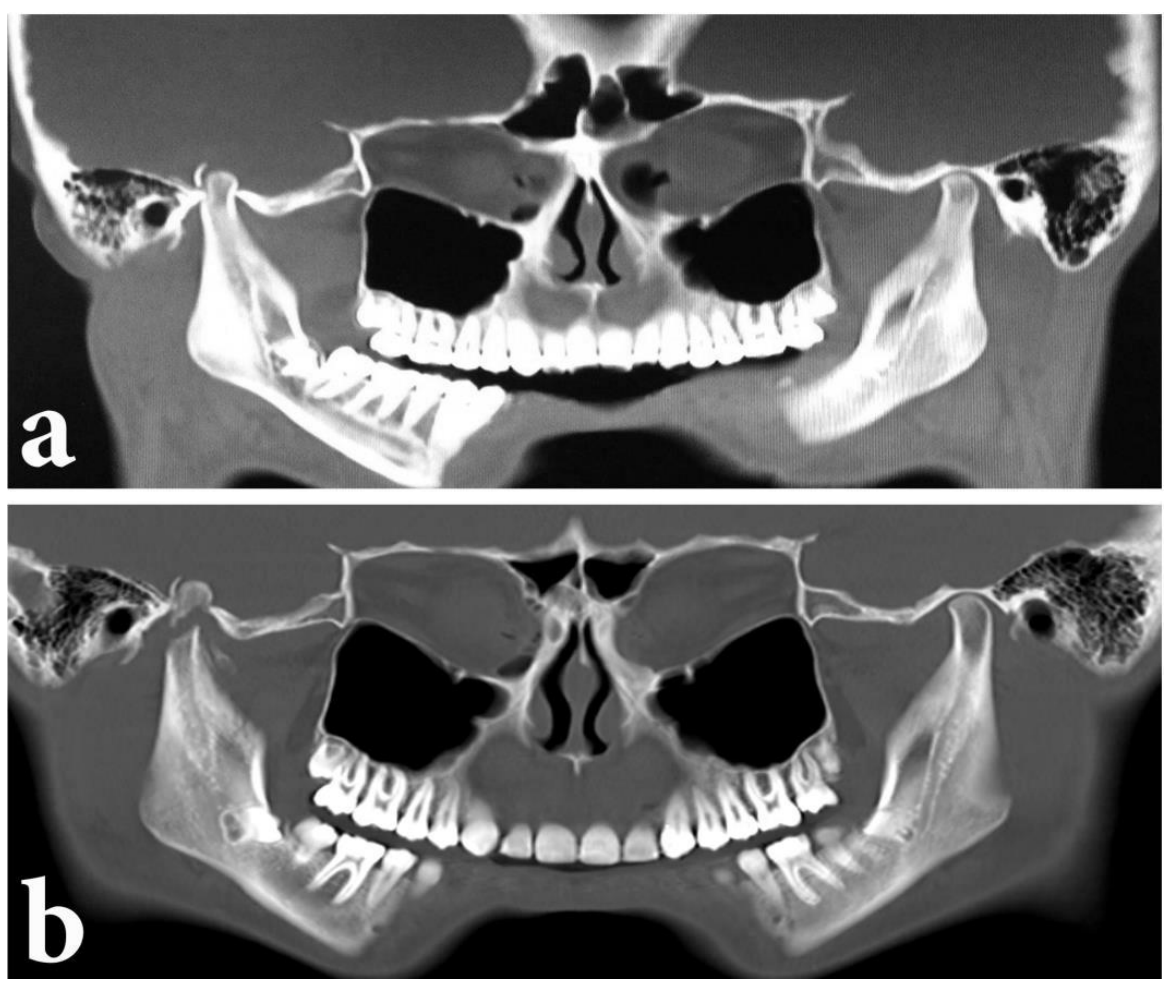

Fig. 2 (a) Coronal view on a computed tomography scan showing penetration of the right mandibular condyle into the middle cranial fossa. (b) The same coronal view was taken 08 months postoperatively and showed a stable skeletal relationship. 


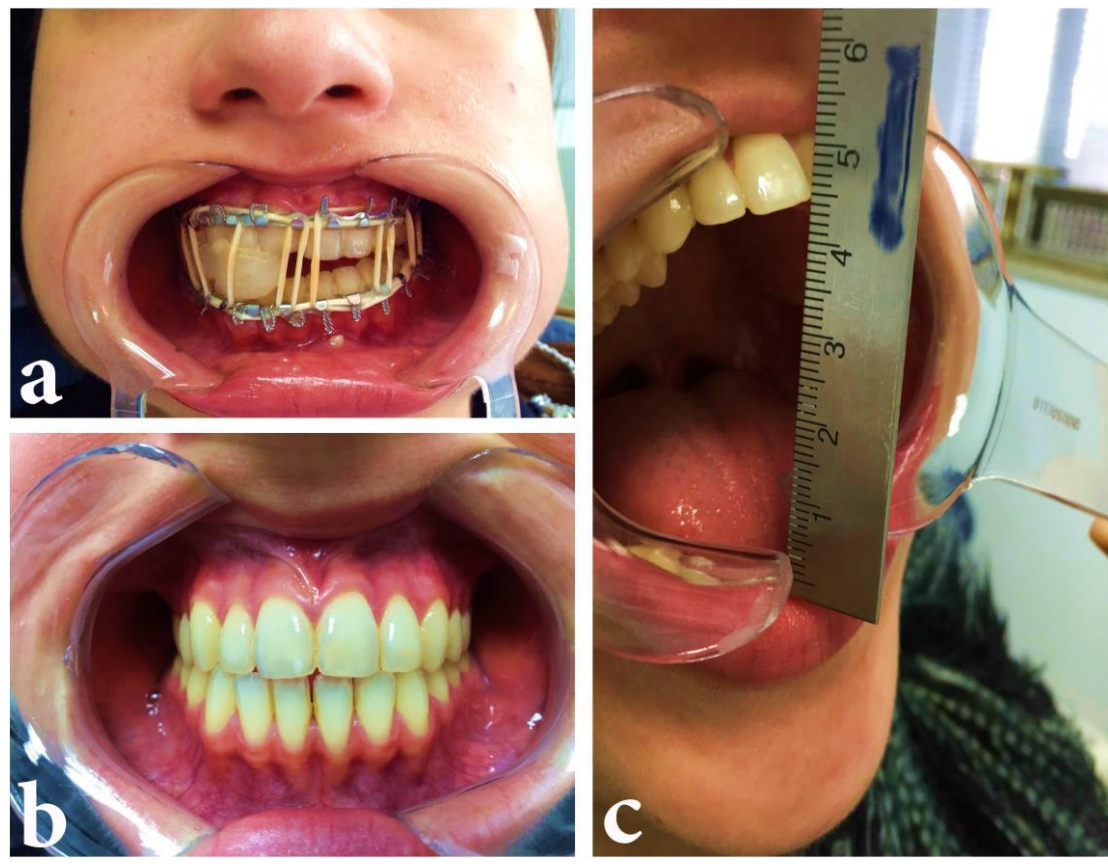

Fig. 3 Patient' pictures: (A) A splint on the right side with elastic intermaxillary traction and fixation; (B) After 60 days the final occlusion position; (C) On the 2 years review, the mouth opening is normal.

\section{CASE REPORT}

A 17-year-old female patient, that had suffered a fall from approximately $1.5 \mathrm{~m}$, was brought to the emergency service complaining of pain in the right pre- auricular area. There were no neurological deficits and the vital signs were stable. On physical examination, there were wounds in the mental area, pre- auricular edema and trismus. A cranial CT (Computed Tomography)-scan was performed, evidencing fracture of the mandible articular portion on the right temporal bone, with superior luxation of the ipsilateral mandible condyle, and associated temporal lobe contusion, evidenced as a hyperdense focal area, and surrounding hypodense halo (Fig 1). The intraoral approach was performed to do an open reduction by condylectomy (Fig 2). In the sequence, intermaxillary fixation was performed with arch bars and with the use of splint, to keep the right side in a dental disocclusion

for 4 weeks (Fig 3). Due to the small size of the parenchymal lesion, and stable neurological scenario, intracranial approach was not necessary. The patient remained asymptomatic afterwards.

\section{DISCUSSION}

Although fractures of the condylar process of the mandible are frequent among all the mandible fractures, condyle dislocation, involving the fracture of the glenoid fossa of the temporal bone, towards the cranial middle fossa has been rarely reported ${ }^{[1,7]}$. In the case of trauma, several anatomical conditions prevent the dislocation of the condyle in the upper direction ${ }^{5,8]}$. Anatomical variations seem to be determinant for this condition to happen ${ }^{[5]}$, since it is present only when the "intrinsic safety mechanisms" fail or are absent ${ }^{[2,4,6,7,9,10]}$. These mechanisms include: 1) the propensity of the thin neck of the condylar process to fracture when exposed to enough strength during trauma, preventing the direct transmission of the force upwards, toward the glenoid fossa; 2) the increased medial-lateral diameter of the condyles, found in the majority of the adults, therefore dissipating the strength of the impact through a wider area inside the fossa; 3) the presence of the posterior teeth, serving as a shock absorber, preventing the superior dislocation of the condyle ${ }^{[1,2,6,7]}$. 
The temporo-mandibular joint is composed by the mandible condyle and the glenoid fossa of the temporal bone. The medial portion of the fossa is relatively is thin, but the lateral component is usually thick and supported by the anterior and posterior roots of the zygomatic bone. The lateral component seems to be the one responsible for blocking the entrance of the condylar process in the middle cranial fossa, with rare exceptions. Since the dislocation is prevented, the forces responsible for the fractures, generally, lead to collapse of the fragile neck of the condylar process ${ }^{[4,5}$.Several authors point anatomical details that can facilitate this type of lesion: absence of posterior teeth, round and small condyle, excess of pneumatization of the temporal bone, sudden impact in the mandible when the mouth is open ${ }^{[5,8]}$.

There is a well-documented predilection for young female patients ${ }^{[3,10,11]}$. Steuwer et al. performed a wide analysis regarding gender and age of the 45 cases previously reported ${ }^{10]}$. There were 27 cases among women, and 18 cases among men. The incidence is even higher in patients younger than 15 years of age (12 cases among girls, and only 3 cases among boys). From 16 to 30 years of age, there were 9 female patients versus 6 male patients, and in the 51 to 70 year-old range, 1 female patient versus 2 male patients.

The mechanism of trauma was meticulously studied on previous reports. High-speed road traffic accidents and falls are the main cause of this type of injury and direct impact on the patient chin seems to be the proposed mechanism. It is understood by observing the anatomic structure of the temporomandibular joint that this pathologic entity rarely occurs. Other factors may reduce the risk of dislocation into the cranium: the mandible usually fractures in subcondylar region due to relative weakness of condylar neck, acting as "safety mechanism". Another preventive factor is meniscus and contiguous muscle structure. Meniscus and its ligaments dissipate the impact force ${ }^{[2,3,6,7,9]}$. Clinically, the diagnosis of the fracture can be missed, since there is sometimes lack of signs and symptoms: pre-auricular pain or hypersensibility, deviation of the mandible ipsilateral to the fracture, contralateral open bite anteriorly and decreased opening or closure of the mouth. Bleeding through the external acoustic meatus is common. On physical examination, the palpation, revealing an empty cavity on the joint is an important sign, indicating the need to perform a CT-scan, that can confirm or exclude the condylar dislocation to the middle cranial fossa ${ }^{[1,4,5]}$.

The low incidence of intracranial mandible condylar dislocation also seems to be related to diagnostic issues. The conventional radiographic methods provide a difficult visualization of the entity, and sometimes the diagnosis is made lately, during the course of the treatment ${ }^{[5,11]}$. This type of lesion is generally undiagnosed due to the articular edema, articular dislocation or subcondilar fractures with poor radiographic findings ${ }^{[1,4,6,10]}$. In addition, mortality due to intracranial lesions caused by condylar dislocation and other concomitant traumatic lesions is extremely rare ${ }^{[4,12]}$.

Among the associated traumatic lesions and neurological complications of this pathology, intracranial hematomas (epidural, subdural, intraparenchymal), cranial nerve palsies, combined with uni or bilateral skull base fractures, ipsilateral loss of hearing due to lesion of the cochlear nerve, CSF-leakage and parenchymal damage, as contusions and concussion, are described ${ }^{[1,13]}$.

The literature suggests a treatment protocol that included: neurosurgical evaluation and CT-scan as part of the diagnosis and approach planning; conservative attempt of reduction of the dislocation under general anesthesia, for those patients without the indication of neurosurgical intervention; and, when necessary, neurosurgical approach with craniotomy, open correction of the condyle dislocation and cranial 
reconstruction. The latter group comprises those patients with associated neurological complications or fracture of the facial bones, delayed diagnosis or treatment, or in those cases with conservative treatment failure ${ }^{[2,14]}$.

Condylectomy and coronoidectomy via an intraoral approach, while leaving the condylar head in the cranial fossa, decreases the risk of secondary brain injury ${ }^{[15]}$, so was chosen as the surgical approach in our case together with intermaxillary fixation and postoperative rehabilitation consisting of jaw opening exercises. The outcome for the patient was ideal, with maximal mouth opening of $45 \mathrm{~mm}$ and mild deviation to the right during opening.

\section{CONCLUSION}

This injury is extremely rare in patients with orofacial trauma. Adequate radiographic examination, including Tomography and Magnetic Resonance, is necessary to obtain the proper diagnosis and to determine an effective management. The risk of intracranial complications on these cases makes a necessary close cooperation in a multidisciplinary approach involving neurosurgery and oral maxillofacial surgery.

\section{CONFLICT OF INTEREST:}

The authors confirm no conflict of interest.

\section{REFERENCES}

[1]. Seymour RL, Irby WB (1976) Dislocation of the condyle into the middle cranial fossa. J Oral Surg 34:180-183.

[2]. Vaezi T, Rajaei SA, Abrishami MH, Taghvaei ME (2014) Dislocation of the mandibular condyle into the middle cranial fossa: a case report. Oral Maxillofac Surg 18:69-73

[3]. Barron RP, Kainulainen VT, Gusenbauer AW, Hollemberg R, Sandor GKB (2002) Fracture of glenoid fossa and traumatic dislocation of mandibular condyle into middle cranial fossa. Oral Surg Oral Med Oral Pathol Oral Radiol Endod 93:640 - 642.

[4]. Harstall R, Gratz KW, Zwahlen RA (2005) Mandibular Condyle Dislocation Into the Middle Cranial Fossa: A Case Report and Review of Literature. J Trauma 59:1495-1503.

[5]. Clauser L, Tieghi R, Polito J, Galie M (2006) Dislocation of the Mandibular Condyle into the
Middle Cranial Fossa. J Craniofacial Surg 17-3: 47-51.

[6]. Menon S, Sinba R (2008) Gap athroplasty for mandibular condyle dislocation and impaction into the middle cranial fossa. J Oral Maxillofac Surg 66:2390- 2393.

[7]. Tutela JP, Verbist DE, Kelishadi S, Little JA (2013) Traumatic Dislocation of the Mandibular Condyle into the Middle Cranial Fossa in an Elderly Patient J Craniofac Surg 24(5):1703-5

[8]. Wendell B, Whitacre MD (1996) Dislocation of the mandibular condyle into the middle cranial fossa: review of the literature and report of a case. Plast Reconstr Surg 38:23-26.

[9]. Taglialatela Scafati C, Aliberti F, Scotto di Clemente S, et al. (2008) Dislocation of a fractured mandibular condyle into the middle cranial fossa: a case treated by an extracranial approach. Childs Nerv Syst 24:1067-1070.

[10]. Struewer J, Kiriazidis I, Fiiel J, Dukatz T, Frangen T, Ziring E (2012) Dislocation of the mandibular condyle into the middle cranial fossa causing and epidural hematoma. $\mathrm{J}$ Craniomaxillofac Surg 40:396-399.

[11]. Kallal RH, Gans BJ, Lagrotteria LB (1977) Cranial dislocation of mandibular condyle. Oral Surg 43:2-10.

[12]. Monteiro JLGC, Arruda JAA, Melo ARS, Barbosa RJV, Carneiro SCAS, Vasconcelos BCE (2019) Updated review of traumatic dislocation of the Mandibular Condyle into the Middle Cranial Fossa. J Oral Maxillofac Surg 77:132.e1-132.e16.

[13]. Sandler NA, Ozaki WH, Ochs MW, Marion DW (1996) Intracranial reduction of an intact mandibular condyle displaced into the middle cranial fossa. J Oral Maxillofac Surg 54:506 510.

[14]. Kroetsch LJ, Brook AL, Kader A, Eisig SB (2001) Traumatic dislocation of the mandibular condyle into the middle cranial fossa: report of a case, review of the literature and a proposal management protocol. J Oral Maxillofac Surg 59:88-94.

[15]. Chen I, Chang CM, Yuan-Chien Chen M, Chen KJ (2019) Traumatic dislocation of the mandibular condyle into the middle cranial fossa treated by an intraoral approach. J Formos Med Assoc 118(7): 1161-1165.

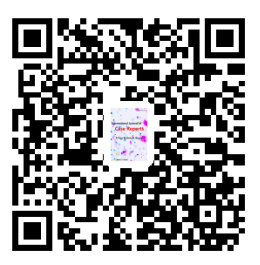

